\title{
Resolution enrichment of side scan sonar image using wavelet based interpolation methods
}

\author{
R. Kumudham ${ }^{1 *}$, S. Tarun ${ }^{2}$, R. Avinash ${ }^{3}$, V. Rajendran ${ }^{4}$ \\ ${ }^{I}$ Department of ECE, School of Engineering, Vels Institute of Science, Technology and Advanced Studies (VISTAS), Chennai. \\ ${ }^{2}$ Department of ECE, School of Engineering, Vels Institute of Science, Technology and Advanced Studies (VISTAS), Chennai. \\ ${ }^{3}$ Department of ECE, School of Engineering, Vels Institute of Science, Technology and Advanced Studies (VISTAS), Chennai. \\ ${ }^{4}$ Department of ECE, School of Engineering, Vels Institute of Science, Technology and Advanced Studies (VISTAS), Chennai \\ *Corresponding author E-mail: kumudham.sree@gmail.com
}

\begin{abstract}
The resolution of the side scan sonar image which is used to detect on seabed such as mines, ship wrecks, etc is low. This paper helps to utilizes image processing techniques to enhance the resolution and thereby it makes detection and classification of underwater objects accurately. The proposed methods discussed in this paper are Discrete wavelet transform and stationary wavelet transform for enhancing the resolution.
\end{abstract}

Keywords: Sonar image, Side scan sonar, resolution, resolution enhancement, object detection, discrete wavelet transform, Stationary wavelet transform, resolution enhancement.

\section{Introduction}

There are many methods to capture the images under the water, of the technique is using side scan sonar equipment since it captures large area. But still the resolution of the side scan images has to be improved, because low frequency wave only reaches the seabed which reduces resolution. Large number of obstacles causes damages to sonar signals such as ambient noise, living habitat, suspended particles this in turn reduces the resolution of the images. Reduction in resolute in degrades the image, so object recognition becomes inaccurate.

\section{Literature survey}

SUNAYA U.SHIRODKAR [1] 2014 proposed interpolation techniques, and lifting wavelet transform. In this paper the proposed method 1 is with respect to spatial resolution where it uses interpolation, stationary wavelet transform and integer wavelet transform. Also another proposed method LWT (Lifting Wavelet Transform) is used for the better resolution of the image. The proposed method is output is compared with existing method to check the result.

U. Anitha and S. Malarkkan ,2014 [2] presented the need of sonar image enhancement and object detection using image fusion techniques. This paper uses DWT (Discrete Wavelet Transform), segmentation and object detection. These algorithms increased the quality of SONAR images. The authors used equipment such as Side scan sonar (SSS) and Synthetic aperture sonar (SAS).

Pingxiang Li, et al[3] used the matching techniques as a method to enhance the resolution of the images .The authors used RG (trigorous working) Algorithm Pixel values of the low resolution images and the enhancement ratio are used to calculate the pixel values of high resolution image. So that the new image has the higher resolution compared to the old image.
Imen Mandhouj et al [4] 2012 discussed about the problem with the object detection on underwater by SONAR images. This is used in many fields such as underwater navigation, seabed mapping, for fishing, for oil min e detection and mines detection. This paper also uses image pre-processing which are classified into two domains spatial domain and transformed domain Mayuri D.Patil [5] reviewed about the techniques of image resolution enhancement. In this paper interpolation, DCT, DFT, DTCWT wavelet transform techniques are reviewed which improves the resolution of the sonar image.

\section{Existing method}

\section{Bilinear interpolation}

The existing work used Bilinear Interpolation Techniques for resolution enhancement. Bilinear interpolation can be used where perfect image transformation is impossible with pixel matching, so that appropriate intensity values are assigned to pixels. Bilinear interpolation uses values of only the 4 nearest pixels, for pixels located in diagonal directions, unlike other interpolation techniques such as nearest neighbour interpolation and bi-cubic interpolation,. Bi-linear interpolation an extension of the linear interpolation is used for interpolating the functions of two variables. The image produced after Bilinear Interpolation has many artifacts like blurring and blocking, etc. Hence wavelet transform methods are used for enhancing the resolution.

\section{Proposed method}

Underwater Images suffer from variations in operating and environmental conditions, target shapes and spatially varying clutter. The researchers developed techniques for underwater image preprocessing, enhancement. It is learnt that the process of getting a high resolved image is yet to be taken up. Hence it is necessary to design a new post processing algorithm for super resolution that is suitable to underwater images. The proposed method involves using wavelet transformation techniques like 
Discrete wavelet transform and Stationary wavelet transform combined together produce high resolution images.

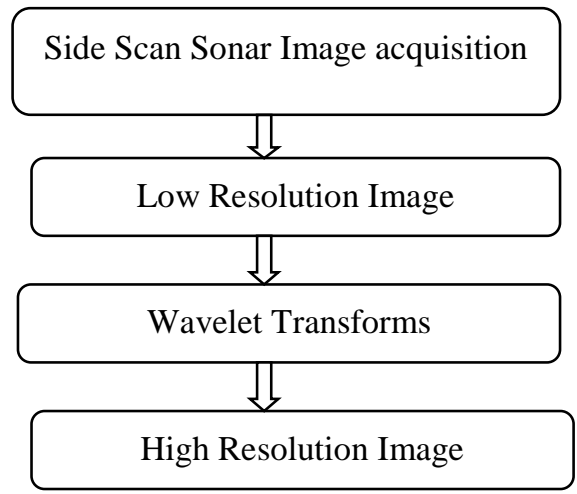

Fig.1: Block diagram of Resolution Enhancement using wavelet transform Wavelet transform has both time and frequency information. It is used in many applications like denoising, compression, etc. One of the main advantage is it preserves information and edges.

\section{Discrete Wavelet Transform (DWT)}

Discrete wavelet transforms divides the signal into set of wavelets which are mutually orthogonal, and where the wavelets samples are discrete in nature. This is one of the main differences from the discrete-time continuous wavelet transform (DT-CWT) and continuous wavelet transform (CWT). The mother wavelet constructed has scaling function describes its scaling properties and shifting function which describes the translation properties. This method is applied to improve the resolution of the side scan sonar image since it preserves edges and reduces noise. When decomposing the image some of the information loss takes place. So the following stationary wavelet transform method is adopted to minimise loss.

\section{Stationary wavelet transform}

Translation invariance in DWT is overcome by using Stationary wavelet transform. Translation-invariance is obtained by removing the up and down samplers in DWT and upsampling the filter coefficients. The SWT is an inherently redundant scheme as the output of each level of SWT contains the same number of samples as the input - so for a decomposition of $\mathrm{N}$ levels there is a redundancy of $\mathrm{N}$ in the wavelet coefficients[6]. The high frequency subbands are interpolated with stationary wavelet transform and the combined with frequency subbands interpolated with discrete wavelet transform. The inverse wavelet transform is obtained to restore image with high resolution.

\section{Results and discussion}

The following fig 2 and fig 3 illustrates the output of bilinear interpolation.

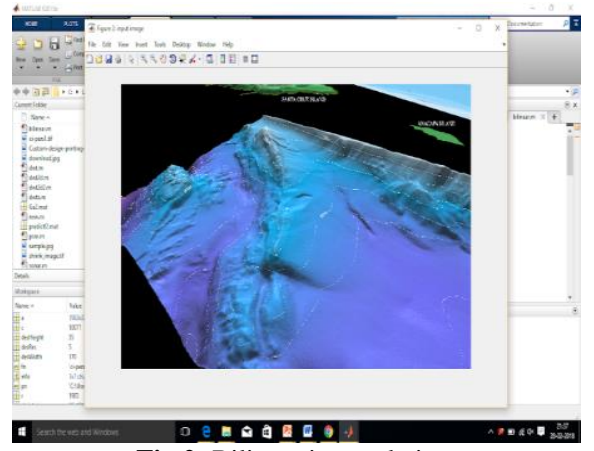

Fig.2: Bilinear interpolation

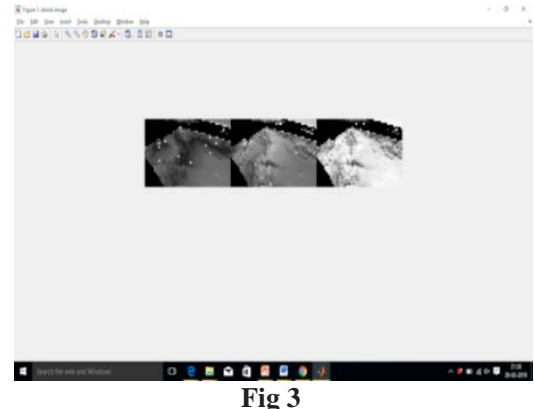

The Following fig 4 illustrates the output of discrete wavelet transform.

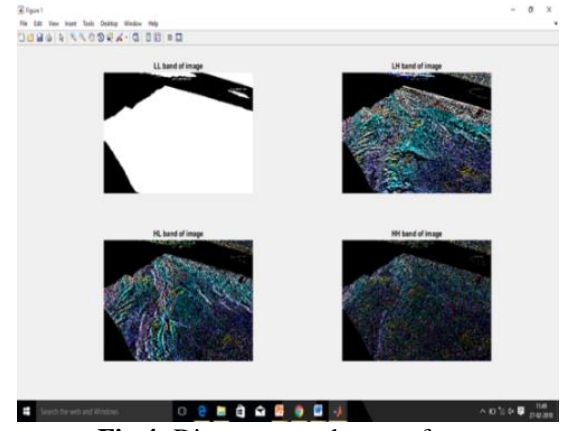

Fig.4: Discrete wavelet transform

\section{Conclusion and future work}

In our proposed work we have enhanced the resolution of sonar images using wavelet transform techniques separately using Discrete wavelet transform and Stationary wavelet transforms and further we get the clear quality of images in high resolution. The future work is planned to improve the resolution of real data images.

\section{References}

[1] Sunaya US, "Image resolution enhancement using various wavelet transforms", International Journal of Advances in Science Engineering and Technology, Vol.1, No.3, (2014).

[2] Anitha U \& Malarkkan S, "Study and Analysis of Sonar Image Enhancement Using Image Fusion Techniques", World Applied Sciences Journal, (2014).

[3] Pingxiang L, Huanfeng S \& Liangpei Z, "A Method of Image Resolution Enhancement Based On The Matching Technique", State Key Laboratory of Information Engineering in Surveying, Mapping and Remote Sensing, Wuhan University, (2012).

[4] Mandhouj I, Amiri H, Maussang F \& Solaiman B, "Sonar image processing for underwater object detection based on high resolution system", 2nd Workshop on Signal and Document Processing, Vol.845, (2012), pp.5-10.

[5] Patil MD \& Khare S, "Overview of techniques used for image resolution enhancement", International Journal on Computer Science and Engineering, Vol.4, No.7, (2012).

[6] Holschneider M, Kronland-Martinet R, Morlet J \& Tchamitchian $\mathrm{P}$, "A real-time algorithm for signal analysis with the help of the wavelet transform", Wavelets, Time-Frequency Methods and Phase Space, (1989), pp. 289-297.

[7] Kumudham R \& Rajendran V, "Side scan sonar image denoising and classification", Journal of Advanced Research in Dynamical \& Control Systems, (2017), pp.55-65.

[8] Kumudham R \& Rajendran V, "Implementation of various segmentation algorithms on side scan sonar images and analysing its performance", $A R P N$, Vol.12, No.8, (2017), pp.2396-2400.

[9] Kumudham R \& Rajendran V, "Speeded up robust feature extraction from underwater sonar images", International Journal of Control Theory and Applications' (IJCTA), Vol.10, No.32, (2017), pp.277-282.

[10] Kumudham R, Aparna SG \& Rajendran V, "Comparison of The Performance Metrics of Median Filter and Wavelet Filter when applied on Sonar Images for Denoising", IEEE sponsored International Conference on Computation of Power Energy Information and Communication, (2012). 